\title{
Thermal Condensate Structure and Cosmological Energy Density of the Universe
}

\author{
Antonio Capolupo, Gaetano Lambiase, and Giuseppe Vitiello \\ Dipartimento di Fisica "E.R. Caianiello", Universitá di Salerno and INFN Gruppo Collegato di Salerno, 84084 Fisciano, Italy \\ Correspondence should be addressed to Antonio Capolupo; capolupo@sa.infn.it
}

Received 13 February 2016; Revised 25 March 2016; Accepted 28 March 2016

Academic Editor: Rong-Gen Cai

Copyright ( 2016 Antonio Capolupo et al. This is an open access article distributed under the Creative Commons Attribution License, which permits unrestricted use, distribution, and reproduction in any medium, provided the original work is properly cited. The publication of this article was funded by $\mathrm{SCOAP}^{3}$.

\begin{abstract}
The aim of this paper is to study thermal vacuum condensate for scalar and fermion fields. We analyze the thermal states at the temperature of the cosmic microwave background (CMB) and we show that the vacuum expectation value of the energy momentum tensor density of photon fields reproduces the energy density and pressure of the CMB. We perform the computations in the formal framework of the Thermo Field Dynamics. We also consider the case of neutrinos and thermal states at the temperature of the neutrino cosmic background. Consistency with the estimated lower bound of the sum of the active neutrino masses is verified. In the boson sector, nontrivial contribution to the energy of the universe is given by particles of masses of the order of $10^{-4} \mathrm{eV}$ compatible with the ones of the axion-like particles. The fractal self-similar structure of the thermal radiation is also discussed and related to the coherent structure of the thermal vacuum.
\end{abstract}

\section{Introduction}

The task of this paper is the analysis of the thermal vacuum condensate for scalar and fermion fields, with specific reference to temperatures characteristic of cosmic microwave background $(\mathrm{CMB})$. The interest in considering the vacuum condensate in relation with $\mathrm{CMB}$ resides in the fact that it is a thermal radiation filling almost uniformly the observable universe and one expects that it plays a relevant role in the universe thermal vacuum structure. CMB appears as a radiation left over from an early stage in the expansion of the universe [1] and has a thermal black body spectrum corresponding to the temperature of $2.72548 \pm 0.00057 \mathrm{~K}$ [2]. The anisotropies contained in the spatial variation in the spectral density are attributed to small thermal variations, presumably generated by quantum fluctuations of matter $[1$, $3,4]$.

In our analysis, we compute the expectation value of the energy momentum tensor density of photon fields on the thermal vacuum. As a result, we obtain the energy density and pressure of the $\mathrm{CMB}$.
Together with the CMB, there is indirect evidence of the existence of the cosmic neutrino background (CNB) which represents the universe's background particle radiation composed of neutrinos (relic neutrinos) [5-9]. The CNB estimated temperature is roughly $1.95 \mathrm{~K}$ [5]. It is therefore interesting to extend our study of thermal vacuum condensate also to the CNB case. Thus, we assume the hierarchical neutrino model and, by computing the energy density of the neutrino thermal vacuum, we check the lower bound of the sum of the active neutrino masses $\sum m_{v}$, which has been estimated from the neutrinos oscillations to be of the order of $0.06 \mathrm{eV}$ [10].

We finally discuss the fractal self-similar structure of the thermal vacuum.

In Section 2, the Thermo Field Dynamics (TFD) formalism is introduced and the general expressions of its energy density and pressure are shown. Explicit computations for Maxwell, scalar, and fermion fields are presented in Section 3 and, in Section 4, the fractal structure of the thermal states is analyzed. Section 5 is devoted to the conclusions. 


\section{Thermal Vacuum and Particle Condensate}

The thermal vacuum state $|0(\theta)\rangle$, with $\theta=\theta(\beta), \beta \equiv 1 /\left(k_{B} T\right)$, and $k_{B}$, the Boltzmann constant, is introduced in the TFD formalism $[11,12]$ in such a way that the thermal statistical average $\mathcal{N}_{a_{\mathbf{k}}}(\theta)$ is given by $\mathcal{N}_{a_{\mathbf{k}}}(\theta)=\left\langle 0(\theta)\left|N_{a_{\mathbf{k}}}\right| 0(\theta)\right\rangle$, with $N_{a_{\mathbf{k}}}=a_{\mathbf{k}}^{\dagger} a_{\mathbf{k}}$, the number operator. The bosonic operators $a_{\mathbf{k}}$ and $a_{\mathrm{k}}^{\dagger}$ have usual canonical commutation relations (CCR).

The explicit form of $|0(\theta)\rangle$ is

$$
|0(\theta)\rangle=\prod_{\mathbf{k}} \frac{1}{\cosh \theta_{k}} \exp \left(\tanh \theta_{k} a_{\mathbf{k}}^{\dagger} b_{\mathbf{k}}^{\dagger}\right)|0\rangle,
$$

and it is recognized to be a two-mode time dependent generalized $\mathrm{SU}(1,1)$ coherent state $[13,14]$, condensate of pairs of $a_{\mathbf{k}}$ and $b_{\mathbf{k}}$ quanta. $|0\rangle$ is the vacuum annihilated by $a_{\mathrm{k}}$ and $b_{\mathrm{k}}$. The auxiliary boson operator $b_{\mathrm{k}}$ commutes with $a_{\mathrm{k}}$ and is introduced in order to produce the trace operation in computing thermal averages. The thermal vacuum $|0(\theta)\rangle$ is normalized to one, $\langle 0(\theta) \mid 0(\theta)\rangle=1, \forall \theta$, and in the infinite volume limit $\langle 0(\theta(\beta)) \mid 0\rangle \rightarrow 0$ as $V \rightarrow \infty, \forall \beta$ (for $\int d^{3} \kappa \theta_{\kappa}$ finite and positive).

One also has $\left\langle 0(\theta(\beta)) \mid 0\left(\theta\left(\beta^{\prime}\right)\right)\right\rangle \rightarrow 0$ as $V \rightarrow \infty, \forall \beta$ and $\beta^{\prime}, \beta^{\prime} \neq \beta$. Thus, $\{|0(\theta(\beta))\rangle\}$ provides a representation of the CCR defined at each $\beta$ and unitarily inequivalent $\forall \beta^{\prime} \neq \beta$ to any other representation $\left\{\left|0\left(\beta^{\prime}\right)\right\rangle\right\}$ in the infinite volume limit.

Note that $a_{\mathbf{k}}$ and $b_{\mathbf{k}}$ do not annihilate the state $|0(\theta)\rangle$. The annihilation operators, say $A_{\mathbf{k}}\left(\theta_{k}\right)$ and $B_{\mathbf{k}}\left(\theta_{k}\right)$, for $|0(\theta)\rangle$, $A_{\mathbf{k}}\left(\theta_{k}\right)|0(\theta)\rangle=0=B_{\mathbf{k}}\left(\theta_{k}\right)|0(\theta)\rangle$, are obtained through the Bogoliubov transformation

$$
\begin{aligned}
& A_{\mathbf{k}}\left(\theta_{k}\right)=e^{i \theta_{k} \mathscr{G}} a_{\mathbf{k}} e^{-i \theta_{k} \mathscr{G}}=a_{\mathbf{k}} \cosh \theta_{k}-b_{\mathbf{k}}^{\dagger} \sinh \theta_{k}, \\
& B_{\mathbf{k}}\left(\theta_{k}\right)=e^{i \theta_{k} \mathscr{G}} b_{\mathbf{k}} e^{-i \theta_{k} \mathscr{G}}=b_{\mathbf{k}} \cosh \theta_{k}-a_{\mathbf{k}}^{\dagger} \sinh \theta_{k},
\end{aligned}
$$

whose generator $\mathscr{G}$ is given by $\mathscr{G}=-i \sum_{\mathbf{k}}\left(a_{\mathbf{k}}^{\dagger} b_{\mathbf{k}}^{\dagger}-a_{\mathbf{k}} b_{\mathbf{k}}\right)$. The thermal vacuum expectation value of the number operator $N_{a_{\mathbf{k}}}=a_{\mathbf{k}}^{\dagger} a_{\mathbf{k}}$ is given by

$$
\mathcal{N}_{a_{\mathbf{k}}}(\theta)=\left\langle 0(\theta)\left|a_{\mathbf{k}}^{\dagger} a_{\mathbf{k}}\right| 0(\theta)\right\rangle=\sinh ^{2} \theta_{k} .
$$

Minimization of the free energy (see below) then leads to the thermal statistical average of $N_{a_{\mathrm{k}}}$

$$
\mathcal{N}_{a_{\mathbf{k}}}^{B}(\theta)=\sinh ^{2} \theta_{k}=\frac{1}{e^{\beta \Omega_{k}}-1},
$$

which is indeed the Bose-Einstein distribution function for $a_{\mathrm{k}}$.

Summing up, the "thermal background" at $T$ is described by the quantum coherent condensate vacuum $|0(\theta)\rangle$, which is the thermal physical vacuum.

We now are ready to compute the contributions of the energy momentum tensor $T^{\mu \nu}$ to the thermal vacuum for Maxwell, scalar, and fermion fields. We observe that the offdiagonal terms of $T^{\mu \nu}$ on the vacuum state are zero for these fields; that is, $\left\langle 0(\theta)\left|T^{i j}(x)\right| 0(\theta)\right\rangle=0$, for $i \neq j$. Therefore, the vacuum condensate is homogenous and isotropic and behaves as a perfect fluid (similar result holds for mixed particles [15-19] and for curved space [20]). Then, the energy density and pressure induced by the condensate (3), at a given time (we consider the red shift $z$ of the universe), can be defined by computing the expectation value of the $(0,0)$ and $(j, j)$ components of the energy momentum tensor of a field on $|0(\theta, z)\rangle$ :

$$
\begin{aligned}
& \rho(z)=g_{00}\left\langle 0(\theta, z)\left|: T^{00}(x):\right| 0(\theta, z)\right\rangle, \\
& \rho(z)=g_{j j}\left\langle 0(\theta, z)\left|: T^{j j}(x):\right| 0(\theta, z)\right\rangle .
\end{aligned}
$$

Here, : $\cdots:$ denotes the normal ordering with respect to $|0\rangle$ and no summation on the index $j$ is intended.

\section{Energy Density of Thermal Vacuum and CMB Temperature}

In the photon fields case, the explicit expression of the energy momentum tensor density $T_{\gamma}^{\mu \nu}$ is $T_{\gamma}^{\mu \nu}=-F^{\mu \alpha} F_{\alpha}^{\nu}+$ $(1 / 4) g^{\mu \nu} F_{\alpha \beta} F^{\alpha \beta}[21,22]$. As usual, $F^{\alpha \beta}=\partial^{\beta} A^{\alpha}-\partial^{\alpha} A^{\beta}\left(g^{\mu \nu}=\right.$ $(1,-1,-1,-1), \mu=0,1,2,3 ; \hbar=1=c$ will be used throughout the paper). The thermal vacuum condensate energy density is then

$$
\rho_{\gamma}(z)=\int d^{3} k \Omega_{k}\left\langle 0(\theta, z)\left|: a_{k}^{\dagger} a_{k}:\right| 0(\theta, z)\right\rangle
$$

where $\Omega_{k}=k$ for photons. The result we obtain is

$$
\rho_{\gamma}(z)=\frac{\pi^{2} k_{B}^{4}(1+z)^{4} T_{\gamma}^{4}}{15 \hbar^{3} c^{3}} .
$$

In a similar way, the contribution given to the pressure by the thermal vacuum condensate of photons field is

$$
p_{\gamma}(z)=\frac{\pi^{2} k_{B}^{4}(1+z)^{4} T_{\gamma}^{4}}{45 \hbar^{3} c^{3}}
$$

The equation of state is then $w_{\gamma}(z)=p_{\gamma}(z) / \rho_{\gamma}(z)=1 / 3$, which is the equation of state of the radiation. Equations (7) and (8) reproduce of course the results obtained by solving the Boltzmann equation for the distribution function of photons in thermal equilibrium [5]. The advantage of the present computation is that the role of the boson condensate in obtaining such a result is underlined. Taking the present $\mathrm{CMB}$ temperature, $T_{\gamma}=2.72548 \pm 0.00057 \mathrm{~K}$, and the present red shift of the universe, $z=0$, one obtains the value of the thermal vacuum energy density, $\rho_{\gamma}=2 \times$ $10^{-51} \mathrm{GeV}^{4}$, which of course coincides with the energy density of the CMB [5].

Leaving apart the photon case, we consider now massive boson and fermion fields. The energy momentum tensor density is given by $T_{B}^{\mu \nu}(x)=\partial_{\mu} \phi(x) \partial^{\mu} \phi(x)-$ $(1 / 2) g_{\mu \nu}\left(\partial^{\rho} \phi(x) \partial_{\rho} \phi(x)-m^{2} \phi(x)^{2}\right)$ for free real scalar fields $\phi$ and $T^{\mu \nu}=(i / 2) \bar{\psi} \gamma^{\mu} \overleftrightarrow{\partial^{v}} \psi$ for free Majorana spinor fields $\psi$ 
At any epoch, the thermal vacuum energy and thermal pressure are given by (5), which in the case of the field $\phi$ give

$$
\begin{aligned}
& \rho_{B}=\frac{1}{2}\left\langle 0(\theta, z)\left|:\left[\pi^{2}(x)+(\vec{\nabla} \phi(x))^{2}+m^{2} \phi^{2}(x)\right]:\right| 0(\theta, z)\right\rangle ; \\
& p_{B}=\left\langle 0(\theta, z)\left|:\left\{\left(\partial_{j} \phi(x)\right)^{2}+\frac{1}{2}\left[\pi^{2}(x)-(\vec{\nabla} \phi(x))^{2}-m^{2} \phi^{2}(x)\right]\right\}:\right| 0(\theta, z)\right\rangle .
\end{aligned}
$$

In the case of the isotropy of the momenta $k_{1}=k_{2}=k_{3}$, these can be written as

$$
\rho_{B}=\int \frac{d^{3} \mathbf{k}}{(2 \pi)^{3}} \Omega_{k}\left\langle 0(\theta, z)\left|a_{\mathbf{k}}^{\dagger} a_{\mathbf{k}}\right| 0(\theta, z)\right\rangle ;
$$

Explicitly, they become

$$
\begin{gathered}
\rho_{B}(z)=\frac{1}{2 \pi^{2}} \int_{0}^{\infty} d k k^{2} \frac{\Omega_{k}}{\exp \left(\Omega_{k} / k_{B} T_{\gamma}(1+z)\right)-1}, \\
p_{B}(z)=\frac{1}{6 \pi^{2}} \int_{0}^{\infty} d k k^{2}\left[\frac{k^{2}}{\Omega_{k}}\right. \\
\cdot \frac{1}{\exp \left(\Omega_{k} / k_{B} T_{\gamma}(1+z)\right)-1}-\left(\frac{k^{2}}{\Omega_{k}}+\frac{3 m^{2}}{2 \Omega_{k}}\right) \\
\left.\cdot \frac{\exp \left(\Omega_{k} / 2 k_{B} T_{\gamma}(1+z)\right)}{\exp \left(\Omega_{k} / k_{B} T_{\gamma}(1+z)\right)-1} \cos \left(2 \Omega_{k} t\right)\right] .
\end{gathered}
$$

Notice that the vacuum energy density at thermal equilibrium $\rho_{B}(z),(12)$, coincides with the result obtained by solving the Boltzmann equation for the particle Bose distribution function [5]. The difference to the pressure $p_{B}(z)$ between the contributions coming from the vacuum condensate and the ones coming solely from the Bose distribution function appears in (13). The second term on the RHS of (13) appears due to the condensate of the physical vacuum contributing with nonvanishing values of $\left\langle 0(\theta, z)\left|a_{\mathbf{k}} a_{-\mathbf{k}}\right| 0(\theta, z)\right\rangle$ and $\left\langle 0(\theta, z)\left|a_{\mathbf{k}}^{\dagger} a_{-\mathbf{k}}^{\dagger}\right| 0(\theta, z)\right\rangle$. Should the vacuum be the trivial one $|0\rangle$, these contributions would be identically zero. To our knowledge, the presence of the time dependent term of RHS of (13) is a new result which has not been reported till now in the literature.

By considering the present epoch, $z=0, T=T_{\gamma}$, and by solving numerically the integral in (12), one has the contribution to the vacuum energy given by $\rho_{B} \simeq 9 \times 10^{-52} \mathrm{GeV}^{4}$ for masses less than or equal to the CMB temperature $m \leq T_{\gamma}$; that is, $m \leq 2.3 \times 10^{-4} \mathrm{eV}$ (e.g., possible candidates are axionlike with $\left.m_{a} \in\left(10^{-3}-10^{-6}\right) \mathrm{eV}\right)$. The maximum value of $\rho_{B}$ is obtained for $m \ll 10^{-4} \mathrm{eV}$. In this case, one has $\rho_{B} \simeq$ $10^{-51} \mathrm{GeV}^{4}$. Negligible values of $\rho_{B}$ are obtained for boson masses $m \gg 10^{-3} \mathrm{eV}$.
In the fermion case, the Fermi-Dirac distribution function is obtained:

$$
\mathcal{N}_{a_{\mathbf{k}}}^{F}(\theta)=\sin ^{2} \theta_{k}=\frac{1}{e^{\beta \Omega_{k}}+1} .
$$

The thermal vacuum contribution to the energy density and to the pressure is

$$
\begin{aligned}
& \rho_{F}=\frac{1}{2}\left\langle 0(\theta, z)\left|:\left[-i \bar{\psi} \gamma_{j} \partial^{j} \psi+m \bar{\psi} \psi\right]:\right| 0(\theta, z)\right\rangle ; \\
& p_{F}=\left\langle 0(\theta, z)\left|:\left(\frac{i}{2} \bar{\psi} \gamma_{j} \overleftrightarrow{\partial_{j}} \psi\right):\right| 0(\theta, z)\right\rangle,
\end{aligned}
$$

respectively. In (15), the relation $(i / 2) \bar{\psi} \gamma_{0} \overleftrightarrow{\partial_{0}} \psi=i \bar{\psi} \gamma_{0} \partial_{0} \psi=$ $-i \bar{\psi} \gamma_{j} \partial^{j} \psi+m \bar{\psi} \psi$ is used. For Majorana fields, (15) and (16) give

$$
\begin{aligned}
& \rho_{F}=\sum_{r} \int \frac{d^{3} \mathbf{k}}{2 \pi^{3}} \Omega_{k}\left\langle 0(\theta, z)\left|\alpha_{k}^{r \dagger} \alpha_{k}^{r}\right| 0(\theta, z)\right\rangle ; \\
& p_{F}=\frac{1}{3} \sum_{r} \int \frac{d^{3} \mathbf{k}}{2 \pi^{3}} \frac{k^{2}}{\Omega_{k}}\left\langle 0(\theta, z)\left|\alpha_{k}^{r \dagger} \alpha_{k}^{r}\right| 0(\theta, z)\right\rangle,
\end{aligned}
$$

where $\alpha_{k}^{r}, r=1,2$, is the annihilator of fermion field.

The explicit expressions of the energy density and pressure are

$$
\begin{aligned}
& \rho_{F}(z)=\frac{1}{\pi^{2}} \int_{0}^{\infty} d k k^{2} \frac{\Omega_{k}}{\exp \left(\Omega_{k} / k_{B} T_{\gamma}(1+z)\right)+1}, \\
& p_{F}(z)=\frac{1}{3 \pi^{2}} \int_{0}^{\infty} d k \frac{k^{4}}{\Omega_{k}} \frac{1}{\exp \left(\Omega_{k} / k_{B} T_{\gamma}(1+z)\right)+1},
\end{aligned}
$$

respectively. These equations coincide with the energy density and pressure obtained by solving the Boltzmann equation for the fermion distribution function [5]. For $z=0$ and masses $m \leq T_{\gamma}$, we find at $T=T_{\gamma}$ the maximum value of $\rho_{F}$, 
that is, $\rho_{F} \sim 1.6 \times 10^{-51} \mathrm{GeV}^{4}$, which is of the same order of $\mathrm{CMB}$ energy. The state equation is $w_{F} \sim 1 / 3$. Condensates of heavier fermions give negligible contributions to the universe energy. Indeed, the number density of nonrelativistic particles $\left(m \gg T_{\gamma}\right)$ is exponentially suppressed by insufficient pair production due to low temperature. Only the condensates of fields with masses less than or equal to $10^{-4} \mathrm{eV}$, for example, neutrinos, may give relevant contributions.

Taking into account such results, from (19) and (20), we compute the energy density and pressure for the thermal vacuum of the three neutrino fields at the cosmic neutrino background (CNB) temperature $T_{v}=1.95 \mathrm{~K}^{1}$

For $z=0$ and neutrino masses $m_{i} \sim 10^{-4} \mathrm{eV}$, the maximum value of the energy density turns out to be $\rho_{\nu} \sim 0.5 \times$ $10^{-51} \mathrm{GeV}^{4}$, with state equation $w_{v} \sim 1 / 3$. The thermal condensate of neutrinos with larger masses would give negligible contributions to $\rho_{\nu}$. Adopting as customary $\rho_{\nu} \leq \rho_{\gamma}$ and taking the mass $m_{\nu, 1} \sim 10^{-4} \mathrm{eV}$ (which leads to $\rho_{\nu} \leq \rho_{\gamma}$ ) by the lighter neutrino mass, one can derive $m_{\gamma, 2}$ and $m_{v, 3}$ from the hierarchical neutrino model and $\Delta m_{12}^{2}=8 \times 10^{-5} \mathrm{eV}^{2}$ and $\Delta m_{23}^{2}=2.7 \times 10^{-3} \mathrm{eV}^{2}$. The result is $m_{\gamma, 2}=9 \times 10^{-3} \mathrm{eV}$ and $m_{v, 3}=5.3 \times 10^{-2} \mathrm{eV}$, and thus $\sum m_{v}=6 \times 10^{-2} \mathrm{eV}$, as it should be in agreement with its estimated lower bound.

We stress that in our discussion we consider the contribution of the condensate neutrino couples to the background energy. In this case, the condensate of neutrinos with larger masses gives negligible contributions to the energy density. Considering instead the particle contribution to the energy budget of the universe, a higher contribution should be obtained by nonrelativistic neutrinos $\left(m_{\nu} \gg T_{\gamma}\right)$, with larger mass.

\section{Fractal Structure of the Thermal States}

Finally, we show that the thermal vacuum $|0(\theta)\rangle$ has a fractal self-similar structure. Let us consider the time dependent case $\theta=\theta(t)$. We will use the notation $|0(\theta(t))\rangle \equiv|0(t)\rangle$. The boson vacuum $|0(t)\rangle$ provides the quantum representation of the system of couples of damped/amplified oscillators [23]

$$
\begin{aligned}
m \ddot{x}+\gamma \dot{x}+\kappa x & =0, \\
m \ddot{y}-\gamma \dot{y}+\kappa y & =0, \\
L & =m \dot{x} \dot{y}+\frac{\gamma}{2}(x \dot{y}-\dot{x} y)-\kappa x y,
\end{aligned}
$$

where "dot" denotes time derivative, $m, \gamma$, and $\kappa$ are positive real constants, and $L$ is the Lagrangian from which (21) and (22) are derived.

To see indeed how $|0(t)\rangle$ is obtained, one proceeds to the canonical quantization of the system described by (21)(23) and assumes that the canonical commutation relations hold, $\left[x, p_{x}\right]=i \hbar=\left[y, p_{y}\right],[x, y]=0=\left[p_{x}, p_{y}\right]$. The corresponding sets of annihilation and creation operators are

$$
\begin{aligned}
& \alpha \equiv\left(\frac{1}{2 \hbar \Omega}\right)^{1 / 2}\left(\frac{p_{x}}{\sqrt{m}}-i \sqrt{m} \Omega x\right) ; \\
& \widetilde{\alpha} \equiv\left(\frac{1}{2 \hbar \Omega}\right)^{1 / 2}\left(\frac{p_{y}}{\sqrt{m}}-i \sqrt{m} \Omega y\right),
\end{aligned}
$$

with $\left[\alpha, \alpha^{\dagger}\right]=1=\left[\widetilde{\alpha}, \widetilde{\alpha}^{\dagger}\right],[\alpha, \widetilde{\alpha}]=0=\left[\alpha, \widetilde{\alpha}^{\dagger}\right]$. The canonical linear transformations $a \equiv(1 / \sqrt{2})(\alpha+\widetilde{\alpha}), b \equiv(1 / \sqrt{2})(\alpha-$ $\widetilde{\alpha})$ are introduced. It is found [23] that the time evolution of the system ground state (the vacuum) leads out of the Hilbert space of the states, and thus the proper quantization setting is the one of the quantum field theory (QFT). One has therefore to consider operators $a_{\mathbf{k}}$ and $b_{\mathbf{k}}$ and their hermitian conjugates, so as to perform, as customary in QFT, the continuum momentum limit (or the infinite volume limit) by use of the relation $\sum_{k} \rightarrow\left(V /(2 \pi)^{3}\right) \int d^{3} k$ at the end of the computations. The Hamiltonian $H$ of the system is found to be [23] $H=H_{0}+H_{I}$, with

$$
\begin{aligned}
& H_{0}=\sum_{\mathbf{k}} \hbar \Omega_{k}\left(a_{\mathbf{k}}^{\dagger} a_{\mathbf{k}}-b_{\mathbf{k}}^{\dagger} b_{\mathbf{k}}\right), \\
& H_{I}=i \sum_{\mathbf{k}} \hbar \Gamma_{k}\left(a_{\mathbf{k}}^{\dagger} b_{\mathbf{k}}^{\dagger}-a_{\mathbf{k}} b_{\mathbf{k}}\right),
\end{aligned}
$$

where $\theta_{k}(t)=\Gamma_{k} t \equiv\left(\gamma_{k} / 2 m\right) t$ has been used for each $k$-mode. The group structure is the one of $\operatorname{SU}(1,1),\left[H_{0}, H_{I}\right]=0$, and the Casimir operator $\mathscr{C}$ is given by $\mathscr{C}^{2}=(1 / 4)\left(a_{\mathrm{k}}^{\dagger} a_{\mathrm{k}}-b_{\mathrm{k}}^{\dagger} b_{\mathrm{k}}\right)^{2}$. The initial condition of positiveness for the eigenvalues of $H_{0}$ is thus protected against transitions to negative energy states. One then finds that the time evolution of the vacuum $|0\rangle$ for $a_{\mathbf{k}}$ and $b_{\mathbf{k}}$ is controlled by $H_{I}$ and given by $|0(\theta(t))\rangle=$ $e^{-i t(H / \hbar)}|0\rangle=e^{-i t\left(H_{I} / \hbar\right)}|0\rangle$ which gives in fact (1).

One also finds that $|0(t)\rangle$ turns out to be a squeezed coherent state characterized by the $q$-deformation of LieHopf algebra and provides a representation of the CCR at finite temperature which is equivalent [23] to the Thermo Field Dynamics representation $\{|0(\beta)\rangle\}[11,12]$. In the limit of quasi-stationary case with $\beta(t)$ slowly changing in time, minimization of the free energy gives again the Bose-Einstein distribution function equation (3).

Indeed, let us now introduce the functional $\mathscr{F}_{a}$ for the $a$-modes

$$
\mathscr{F}_{a} \equiv\left\langle 0(t)\left|\left(H_{a}-\frac{1}{\beta} \mathcal{S}_{a}\right)\right| 0(t)\right\rangle,
$$

where $H_{a}$ is the free Hamiltonian relative to the $a$-modes, $H_{a}=\sum_{\mathbf{k}} \hbar \Omega_{k} a_{\mathbf{k}}^{\dagger} a_{\mathbf{k}}$, and $\mathcal{S}_{a}$ is given by

$$
\mathcal{S}_{a} \equiv-\sum_{\mathbf{k}}\left\{a_{\mathbf{k}}^{\dagger} a_{\mathbf{k}} \ln \sinh ^{2}(\theta)-a_{\mathbf{k}} a_{\mathbf{k}}^{\dagger} \ln \cosh ^{2}(\theta)\right\} .
$$

Inspection of (26) and (27) suggests that $\mathscr{F}_{a}$ and $\mathcal{S}_{a}$ can be considered as free energy and the entropy, respectively. Minimization of the functional $\mathscr{F}_{a}, \partial \mathscr{F}_{a} / \partial \theta_{k}(t)=0, \forall k$ $[11,12]$ (we consider $\hbar=c=1$ ), then leads to (4) which is the Bose-Einstein distribution function for $a_{\mathbf{k}}$. The first principle of thermodynamics at constant temperature can be then expressed as

$$
d \mathscr{F}_{a}=d \mathscr{E}_{a}-\frac{1}{\beta} \mathcal{S}_{a}=0
$$

where the change in time of the particle condensed in the vacuum turns out to heat dissipation $d Q=(1 / \beta) d \mathcal{S}$ :

$$
d \mathscr{E}_{a}=\sum_{\mathbf{k}} \hbar \Omega_{\mathbf{k}} \dot{\mathcal{N}}_{a}^{\mathbf{k}}(t) d t=\frac{1}{\beta} d \mathcal{S}=d Q,
$$

where $\dot{\mathcal{N}}_{a}^{\mathbf{k}}(t)$ denotes the time derivative of $\mathscr{N}_{a}^{\mathbf{k}}(t)$. 
We now remark that the system of (21) and (22) possesses self-similarity properties. To see this, let us put

$$
\begin{aligned}
& \frac{1}{2}\left[z_{1}(t)+z_{2}^{*}(-t)\right]=x(t), \\
& \frac{1}{2}\left[z_{1}^{*}(-t)+z_{2}(t)\right]=y(t)
\end{aligned}
$$

with $z_{1}(t)=r_{0} e^{-i \Omega t} e^{-\Gamma t}$ and $z_{2}(t)=r_{0} e^{+i \Omega t} e^{+\Gamma t}, \Gamma \equiv \gamma / 2 m$ and $\Omega^{2}=(1 / m)\left(\kappa-\gamma^{2} / 4 m\right), \kappa>\gamma^{2} / 4 m$. Then, we see that (21) and (22) can be rewritten as [24]

$$
\begin{aligned}
& m \ddot{z}_{1}+\gamma \dot{z}_{1}+\kappa z_{1}=0, \\
& m \ddot{z}_{2}-\gamma \dot{z}_{2}+\kappa z_{2}=0 .
\end{aligned}
$$

Solutions of (31) and (32) are in fact $z_{1}(t)=r_{0} e^{-i \Omega t} e^{-\Gamma t}$ and $z_{2}(t)=r_{0} e^{+i \Omega t} e^{+\Gamma t}$ and they describe the parametric time evolution of clockwise and the anticlockwise logarithmic spirals, $r=r_{0} e^{-d \alpha}$ and $r=r_{0} e^{d \alpha}$, with $\alpha(t)=\Gamma t / d$ and $\Omega t=$ $\Gamma t / d=\alpha(t)[24]$.

Thus, (21) and (22) (or equivalently (31) and (32)), whose quantum representation is provided by $|0(t)\rangle$, are found to describe the self-similar fractal structure of their logarithmic spiral solutions $[25,26]$. This establishes the link between the $\mathrm{SU}(1,1)$ coherent states and fractal-like self-similarity [24]. The relation of the photon energy momentum tensor $T_{\gamma}^{\mu \nu}$ with (21) and (22) can also be shown. For details, see [24]. Similar discussions can be done for the fermion vacuum.

\section{Conclusions}

We have studied the thermal vacuum structure at the temperature of the CMB. In the framework of TFD, the energy momentum tensor density of photon has expectation value on the vacuum which agrees with the energy density and pressure of the CMB. In the case of neutrinos and thermal states at the temperature of the CNB consistency has been verified with the estimated lower bound of the sum of the active neutrino masses. The fractal self-similar structure of the thermal vacuum has been also discussed.

\section{Competing Interests}

The authors declare that there are no competing interests regarding the publication of this paper.

\section{Acknowledgments}

Partial financial support from MIUR is acknowledged.

\section{Endnotes}

1. The relic neutrino temperature $T_{v}$ is related to the one of $\mathrm{CMB} T_{\gamma}$ by the relation [5]

$$
\left(\frac{T_{\gamma}}{T_{\gamma}}\right)=\left(\frac{4}{11}\right)^{1 / 2} .
$$

This implies that since, at the present epoch, $T_{\gamma}=$ $2.725 \mathrm{~K}$, one obtains $T_{v}=1.95 \mathrm{~K}$.

\section{References}

[1] E. Gawiser and J. Silk, "The cosmic microwave background radiation," Physics Report, vol. 333-334, no. 4-6, pp. 245-267, 2000.

[2] D. J. Fixsen, "The temperature of the cosmic microwave background," The Astrophysical Journal, vol. 707, no. 2, pp. 916-920, 2009.

[3] C. L. Bennett, A. J. Banday, K. M. Górski et al., "Four-year $C O B E^{*}$ DMR cosmic microwave background observations: maps and basic results," The Astrophysical Journal Letters, vol. 464, no. 1, p. L1, 1996.

[4] P. de Bernardis, P. A. R. Ade, J. J. Bock et al., "A flat Universe from high-resolution maps of the cosmic microwave background radiation," Nature, vol. 404, pp. 955-959, 2000.

[5] S. Weinberg, Cosmology, Oxford University Press, Oxford, UK, 2008.

[6] S. Bashinsky and U. Seljak, "Signatures of relativistic neutrinos in CMB anisotropy and matter clustering," Physical Review D, vol. 69, no. 8, Article ID 083002, 35 pages, 2004.

[7] G. Mangano, G. Miele, S. Pastor et al., "Relic neutrino decoupling including flavour oscillations," Nuclear Physics B, vol. 729, no. 1-2, pp. 221-234, 2005.

[8] E. Komatsu, "Seven-year Wilkinson Microwave Anisotropy Probe (WMAP ${ }^{*}$ ) observations: cosmological interpretation," The Astrophysical Journal Supplement Series, vol. 192, no. 2, p. 18, 2011.

[9] E. Kolb and M. Turner, The Early Universe, Frontiers in Physics, Westview Press, 1994.

[10] M. C. Gonzalez-Garcia, M. Maltoni, J. Salvado, and T. Schwetz, "Global fit to three neutrino mixing: critical look at present precision," Journal of High Energy Physics, vol. 2012, article 123, 2012.

[11] Y. Takahashi and H. Umezawa, "Thermo field dynamics," Collective Phenomena, vol. 2, pp. 55-80, 1975, Reprinted in: International Journal of Modern Physics B, vol. 10, pp. 1755-1805, 1996.

[12] H. Umezawa, Advanced Field Theory: Micro, Macro, and Thermal Physics, AIP, New York, NY, USA, 1993.

[13] A. Perelomov, Generalized Coherent States and Their Applications, Springer, Berlin, Germany, 1986.

[14] J. R. Klauder and B. Skagerstam, Coherent States, World Scientific, Singapore, 1985.

[15] A. Capolupo, S. Capozziello, and G. Vitiello, "Neutrino mixing as a source of dark energy," Physics Letters, Section A: General, Atomic and Solid State Physics, vol. 363, no. 1-2, pp. 53-56, 2007.

[16] A. Capolupo, S. Capozziello, and G. Vitiello, "Dark energy and particle mixing," Physics Letters A, vol. 373, no. 6, pp. 601-610, 2009.

[17] A. Capolupo, S. Capozziello, and G. Vitiello, "Dark energy, cosmological constant and neutrino mixing," International Journal of Modern Physics A, vol. 23, no. 31, pp. 4979-4990, 2008.

[18] M. Blasone, A. Capolupo, S. Capozziello, S. Carloni, and G. Vitiello, "Neutrino mixing contribution to the cosmological constant," Physics Letters, Section A: General, Atomic and Solid State Physics, vol. 323, no. 3-4, pp. 182-189, 2004.

[19] A. Capolupo, M. Di Mauro, and A. Iorio, "Mixing-induced spontaneous supersymmetry breaking," Physics Letters A: General, Atomic and Solid State Physics, vol. 375, no. 39, pp. 34153418, 2011.

[20] F. D. Albareti, J. A. R. Cembranos, and A. L. Maroto, "Vacuum energy as dark matter," Physical Review D, vol. 90, no. 12, Article ID 123509, 2014. 
[21] S. S. Schweber, An Introduction to Relativistic Quantum Field Theory, Harper and Row, New York, NY, USA, 1961.

[22] J. Leite Lopez, Gauge Field Theories: An Introduction, Pergamon Press, Oxford, UK, 1981.

[23] E. Celeghini, M. Rasetti, and G. Vitiello, "Quantum dissipation," Annals of Physics, vol. 215, no. 1, pp. 156-170, 1992.

[24] G. Vitiello, "Fractals, coherent states and self-similarity induced noncommutative geometry," Physics Letters. A, vol. 376, no. 37, pp. 2527-2532, 2012.

[25] H. O. Peitgen, H. Jürgens, and D. Saupe, Chaos and Fractals: New Frontiers of Science, Springer, Berlin, Germany, 1986.

[26] A. A. Andronov, A. A. Vitt, and S. E. Khaikin, Theory of Oscillators, Dover, Mineola, NY, USA, 1966. 

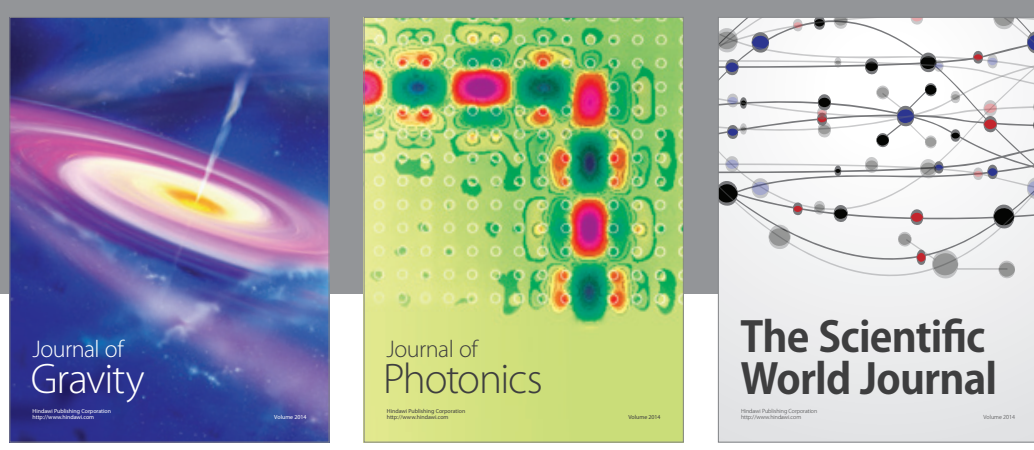

The Scientific World Journal
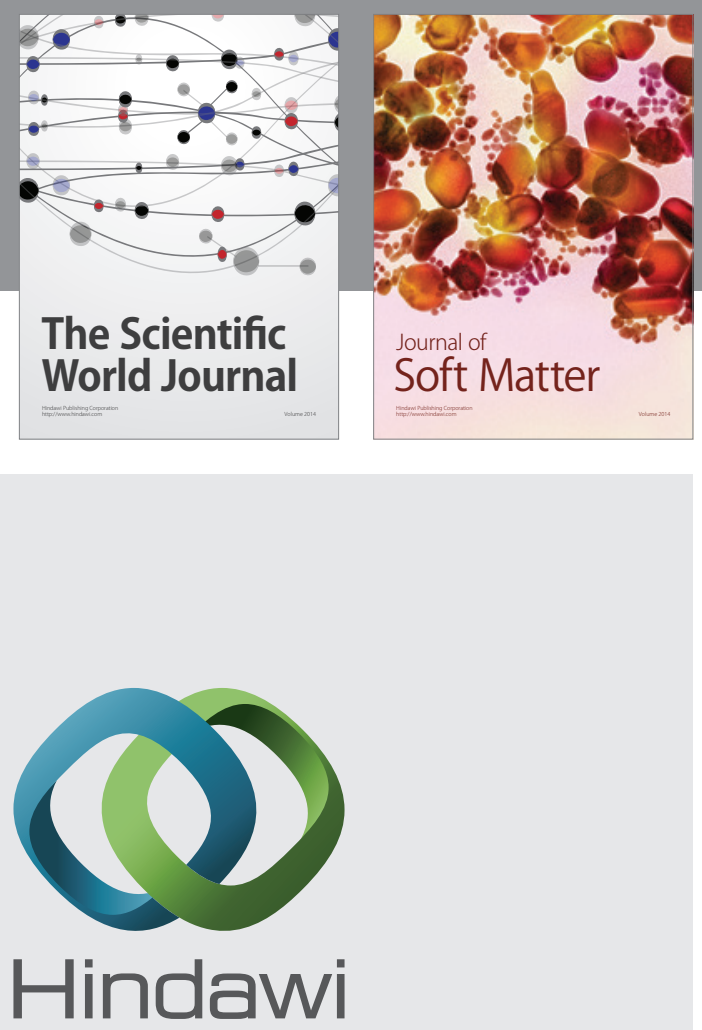

Submit your manuscripts at

http://www.hindawi.com

nternational Journal of

Statistical Mechanics
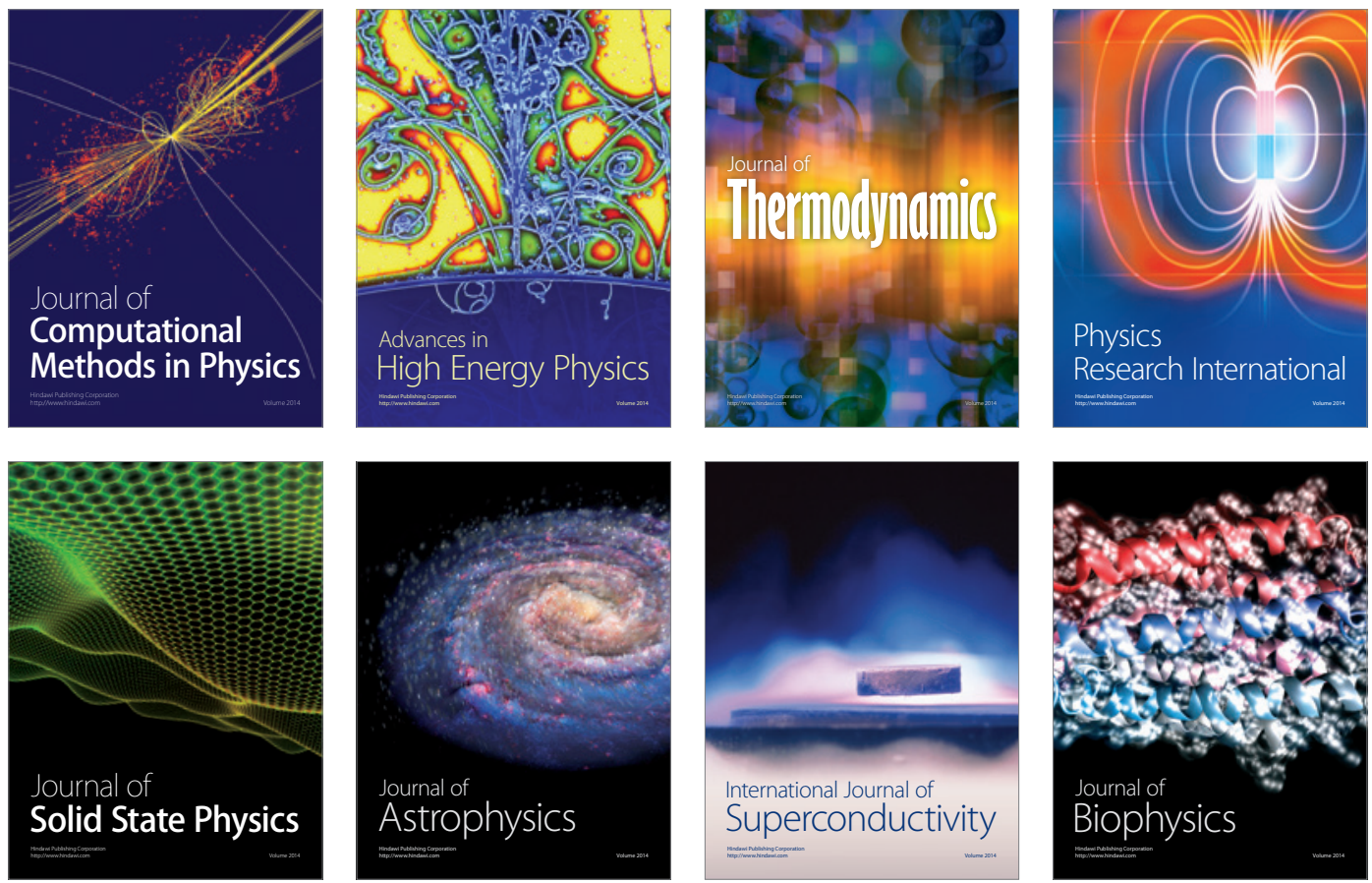
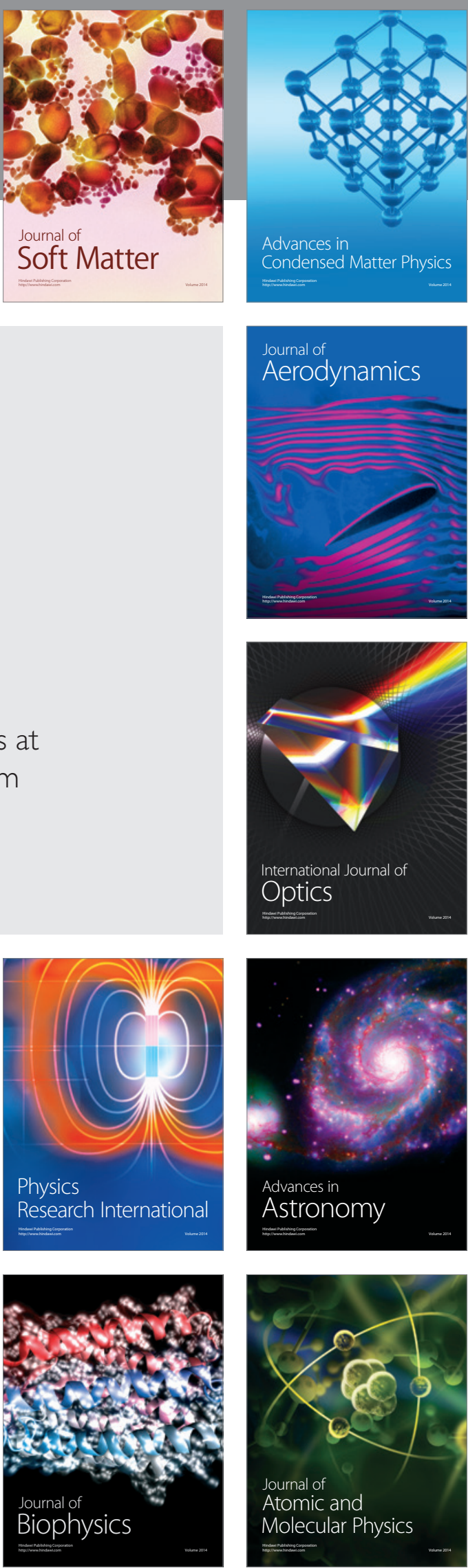